\title{
Secuencia de abordaje diagnóstico-terapéutico para el paciente potencialmente intoxicado. Parte 1: diagnóstico
}

\author{
Jorge G. Pérez-Tuñón, Mayré I. Bautista-Albiter*, Herminio Terán-Flores, Juan C. Pérez-Hernández, \\ José Padilla-Ochoa y Mireille D. Arango-Mathieu \\ Centro Toxicológico, Hospital Ángeles Lomas, Huixquilucan, Ciudad de México, México
}

\begin{abstract}
Resumen
El médico de urgencias con frecuencia atiende a pacientes potencialmente intoxicados, los cuales generalmente representan del 1 al 3\% del total de sus pacientes. Dichos pacientes presentan muy diversos cuadros clínicos dependiendo de las propiedades fisicoquímicas del agente o los agentes involucrados, así como de otros factores, entre los que destacan: el tiempo de evolución, la vía de exposición, el estado de salud previo y la edad del paciente, de tal modo que el abordaje de un paciente expuesto a una sustancia potencialmente tóxica involucra muchos aspectos que resultan vitales y que podrían ser omitidos en caso de no seguir una secuencia que permita la recopilación de toda la información disponible en el menor tiempo posible. Es por ello que en el presente trabajo desarrollamos una secuencia de abordaje diagnóstico-terapéutica para el paciente potencialmente intoxicado, la cual debido a su extensión se ha dividido en dos partes: diagnóstico y tratamiento.
\end{abstract}

Palabras clave: Intoxicación. Toxicología. Sobredosis. Exploración física.

\section{Secuence of diagnostic-therapeutic approach for the potentially intoxicated patient.} Part 1: Diagnosis

\begin{abstract}
The emergency physician frequently cares for potentially poisoned patients, who generally represent 1 to $2 \%$ of his total patients. These patients have very diverse presentations depending on the physico-chemical properties of the agent or agents involved, as well as other factors among which stand out: the time of evolution, the route of exposure, the previous state of health and the age of the patient, so that the approach to a patient exposed to a potentially toxic substance involves many aspects that are vital and could be omitted in case of not following a sequence that allows the collection of all the available information in the shortest possible time. It is because of that in the present work we develop a sequence of diagnostic-therapeutic approach for the potentially intoxicated patient, which due to its extension has been divided into two parts: diagnosis and treatment.
\end{abstract}

Key words: Poisoning. Toxicology. Overdose. Physical examination.

\section{Correspondencia:}

*Jorge G. Pérez-Tuñón

E-mail: toxicología_angeles@yahoo.com.mx
Disponible en internet: 13-09-2021 Rev Educ Investig Emer. 2021;3(3):140-146 www.medicinadeemergencias.com
Fecha de aceptación: 11-05-2021 DOI: 10.24875/REIE.21000020 bajo la licencia CC BY-NC-ND (http://creativecommons.org/licenses/by-nc-nd/4.0/). 


\section{Introducción}

El médico de urgencias con frecuencia atiende a pacientes intoxicados o que han estado expuestos a xenobióticos diversos, lo cual les hace susceptibles de desarrollar una intoxicación. Este tipo de pacientes suele representar entre el 1 y el $3 \%$ del total de pacientes que acuden a los servicios de urgencias, aunque en algunos estudios llevados a cabo en hospitales pediátricos, la cifra ha llegado a superar el 10\% de los ca$\operatorname{sos}^{1-4}$. Cuando ello ocurre, es importante considerar que la forma clínica de presentación suele ser muy variada dependiendo de múltiples factores, entre los que se destacan las propiedades fisicoquímicas del agente o los agentes involucrados, así como del tiempo de evolución, la vía de exposición, el estado de salud previo y la edad del paciente, quien puede acudir asintomático o manifestar un cuadro gastrointestinal, neurológico, respiratorio o hemodinámico, entre muchas otras posibilidades. Teniendo en cuenta lo anterior, el abordaje de un paciente expuesto a una sustancia potencialmente tóxica involucra muchos aspectos que resultan vitales y que podrían ser omitidos en caso de no seguir una secuencia que permita la recopilación de toda la información disponible en el menor tiempo posible, con la finalidad de obtener un diagnóstico acertado y con ello iniciar un plan terapéutico oportuno y efectivo, pues el curso clínico de la intoxicación estará determinado en la mayoría de las veces por el conjunto de acciones que el personal de la salud lleve a cabo en las primeras horas que siguen a la exposición ${ }^{5-8}$.

Con base en lo anterior hemos desarrollado una secuencia de abordaje diagnóstico-terapéutico para el paciente potencialmente intoxicado, la cual permite de forma didáctica contemplar todos los puntos indispensables en lo concerniente a estos pacientes (Tabla 1). No obstante, debemos señalar que el orden planteado busca priorizar los aspectos clave en el abordaje y no indicar una secuencia cronológica obligada que pudiera retrasar las medidas terapéuticas, de tal modo que de manera óptima puedan llevarse a cabo simultáneamente, como cuando se interroga al paciente al tiempo que se obtienen muestras para análisis clínicos, o cuando se le administra el antídoto mientras se le prepara para recibir un método extracorpóreo de eliminación.

\section{Estabilización}

La estabilización consiste en llevar a cabo las medidas necesarias para mantener con vida y reducir la
Tabla 1. Secuencia de abordaje diagnóstico-terapéutico para el paciente potencialmente intoxicado*

\begin{tabular}{|l|l|}
\hline 0 & Estabilización \\
\hline 1 & Interrogatorio \\
\hline 2 & Exploración física \\
\hline 3 & Complemento diagnóstico (laboratorio, gabinete) \\
\hline 4 & Descontaminación \\
\hline 5 & Eliminación \\
\hline 6 & Antídoto \\
\hline
\end{tabular}

*El presente abordaje deberá ajustarse a las necesidades individuales de cada paciente (no todos requieren descontaminación o eliminación ni existen antídotos para todos los xenobióticos).

morbilidad del paciente que es llevado al hospital en estado crítico. Queda claro que en algunas ocasiones el paciente acude por su propio pie, sin presentar deterioro clínico significativo o incluso antes de desarrollar sintomatología, sin embargo, en otras ocasiones puede presentarse en condiciones que comprometan su supervivencia o desarrollarlas en el curso de su abordaje clínico. Es por tal motivo que a esta sección se le ha asignado el número 0 , ya que pueden ser requeridas maniobras de estabilización en cualquier momento durante el curso de una intoxicación. Entre las condiciones que requieren una intervención inmediata podemos destacar: el paro cardiorrespiratorio, el estado de choque, el deterioro neurológico, la dificultad respiratoria severa, el estatus epiléptico y la hipertermia, debido a que representan un riesgo de muerte 0 daño permanente a corto plazo. Por lo anterior, en un paciente de estas características resulta primordial iniciar la reanimación en caso de que así lo requiera, así como asegurar la vía aérea, instalar un acceso venoso, mantener una tensión arterial perfusora, iniciar control de la temperatura corporal y mantener las demás constantes vitales dentro de parámetros que le permitan las mejores condiciones de supervivencia, incluso antes de iniciar el abordaje diagnóstico orientado a toxicología. En este sentido, el monitoreo del paciente aportará información dinámica y sustancial respecto a parámetros como el ritmo cardiaco, los signos vitales o la saturación de oxígeno, debiendo además obtener estudios a la cabecera del paciente como la glucemia capilar, la gasometría o el electrocardiograma, los cuales es posible obtener en poco tiempo y brindan un amplio panorama respecto de sus condiciones generales $^{5-10}$. Con base en ello, la nemotecnia ABCDE (Tabla 2) para el paciente intoxicado pretende señalar 
Tabla 2. ABCDE del paciente intoxicado (diagnóstico-terapéutico)

\begin{tabular}{|c|c|c|}
\hline A & \multicolumn{2}{|l|}{ Vía aérea permeable } \\
\hline B & \multicolumn{2}{|l|}{ Ventilación } \\
\hline C & \multicolumn{2}{|l|}{ Circulación* } \\
\hline D & Dextrostix (glucemia capilar) & Descontaminación \\
\hline $\mathrm{E}$ & Electrocardiograma & Eliminación \\
\hline
\end{tabular}

los principales aspectos a considerar en la estabilización de pacientes críticamente enfermos.

\section{Interrogatorio}

El conocimiento de la historia clínica por parte del médico es fundamental para establecer el diagnóstico y determinar un plan terapéutico. La nemotecnia SPAM nos puede proporcionar en forma rápida un panorama general sobre el paciente, con la cual interrogamos acerca de los signos y síntomas que ha presentado (S), antecedentes personales relevantes $(P)$, alergias $(A)$ y medicamentos $(\mathrm{M})$ que consume normalmente el paciente 0 a los que pudo haber estado expuesto recientemente ${ }^{11}$.

No obstante, para establecer la causa generalmente se requiere un interrogatorio más amplio, el cual gira en torno a tres grandes vertientes: el paciente, el xenobiótico y el entorno. En lo que respecta al paciente, es importante conocer los aspectos que pueden influir en la farmacocinética del xenobiótico, tal es el caso del sexo, la edad y el estado de gravidez (en mujeres), así como interrogar sobre las condiciones de salud previas a la exposición, si el xenobiótico le pertenece como parte de un tratamiento médico (medicamentos) o nunca ha estado expuesto a tal sustancia, así como si tiene historia de depresión o intentos suicidas que puedan explicar la intencionalidad de la exposición.

Entre los aspectos propios del xenobiótico es importante que podamos responder a las interrogantes ¿qué?, ¿cuánto?, ¿cuándo?, ¿por dónde? y ¿por qué? En condiciones ideales se contará con el nombre de este, debiendo averiguar además la cantidad y gramaje (presentación), si se trata de una exposición aguda o crónica y de ser aguda se debe interrogar la hora exacta de la exposición, así como la vía de contacto (oral, cutánea, oftálmica, etc.). No obstante, en muchas ocasiones no se cuenta con el nombre comercial ni el de la sustancia activa, en cuyo caso es importante conocer las características físicas del compuesto y su aplicación (para qué se usa), lo que puede orientar respecto a su toxicidad (analgésicos vs. antibióticos, jabones vs. cáusticos, alcoholes vs. otros hidrocarburos, etc.). A este respecto, cuando se trata de químicos de uso doméstico o industrial, deben ser considerados su estado físico, concentración y propiedades fisicoquímicas como el color, olor, sabor, pH y solubilidad, entre otras. Por otro lado, si se trata de un animal venenoso, una planta o un hongo, quien responde al interrogatorio comúnmente desconoce el nombre de la especie, en cuyo caso la descripción de las características físicas puede ser de utilidad. No obstante, es importante señalar que en ningún caso se retrasarán las acciones terapéuticas en espera de la identificación del xenobiótico, considerando siempre la premisa de «tratar al paciente siempre antes que al tóxico».

Finalmente, es importante interrogar sobre el entorno donde se encontraba el paciente, si existían olor a gas, otras víctimas, cajas o frascos de medicamento, botellas de alcohol u otras sustancias, parafernalia de drogas de abuso, restos de vómito, etc. En este sentido, suelen ser fuentes adecuadas de información los familiares y los paramédicos, y cuando sea posible se enviará a un familiar a recabar mayor información al sitio donde se encontraba el paciente, ya que en ocasiones una segunda búsqueda puede aportar el nombre del xenobiótico mediante el hallazgo del envase vacío o xenobióticos que no se habían considerado previamente ${ }^{5,7,9,12,13}$.

\section{Exploración física}

\section{Toxíndromes}

En 1974, Mofenson y Greensher se dieron a la tarea de agrupar las sustancias tóxicas por la similitud de manifestaciones clínicas que presenta un paciente al estar expuesto a ellas, dicha similitud está relacionada a una interacción común con determinado neurotransmisor y/o sus receptores. A este conjunto de signos y síntomas se les llamó «síndromes tóxicos» 0 «toxíndromes» ${ }^{14}$. La integración de un toxíndrome permite al clínico instaurar un tratamiento específico incluso sin haber obtenido la identificación precisa del xenobiótico, basándose únicamente en parámetros clínicos (Tabla 3). Cabe señalar que en la mayoría de las veces no se presentarán todas las manifestaciones descritas para cada toxíndrome, así como que en algunos casos dichas manifestaciones podrán estar modificadas si se 
Tabla 3. Toxíndromes



presenta una exposición crónica o una que involucre a más de una sustancia. Los principales toxíndromes y sus causas se enlistan a continuación:

- Simpaticomimético. Se debe a una actividad incrementada de las diferentes familias de receptores de adrenalina $\left(\alpha_{1}, \alpha_{2}\right.$ y $\left.\beta\right)$, siendo los xenobióticos más frecuentemente involucrados: epinefrina, norepinefrina, dopamina, aminofilina, teofilina, teobromina, cafeína, efedrina, pseudoefedrina, cocaína, anfetamina, metanfetamina, metilendioximetanfetamina (éxtasis) y otros derivados anfetamínicos; xenobióticos con predominio $\alpha$-adrenérgico: fenilefrina, ergotamina, ergonovina, oximetazolina, nafazolina y tetrahidrozolina; de predominio $\beta$-adrenérgico: salbutamol, salmeterol, clembuterol e isoproterenol.

- Anticolinérgico. Ocurre como consecuencia de la inactividad de la familia de receptores muscarínicos de acetilcolina. Los xenobióticos implicados incluyen: atropina, antidepresivos cíclicos, antihistamínicos de primera generación, antiparkinsonianos, antipsicóticos, carbamazepina, ipratropio y plantas de los géneros Atropa, Datura, Solandra y Brugmancia, entre otras.

- Colinérgico. Se presenta cuando existe un contacto sostenido de la acetilcolina con sus receptores (muscarínicos y nicotínicos), lo cual puede estar relacionado con la exposición a insecticidas organofosforados o carbamatos, a carbamatos de uso médico como fisostigmina o a agentes nerviosos como el sarín, el tabún, el VX o el somán.

- Opioide. Se debe a un agonismo sostenido de los receptores opioides. Dicho agonismo puede estar dado por opiáceos como morfina, codeína, tebaína o papaverina; así como por opioides semisintéticos como buprenorfina, oxicodona, heroína o nalbufina y por opioides sintéticos como fentanilo, tramadol o dextrometorfano, entre otros compuestos.

- Sedante-hipnótico. Es consecuencia de la estimulación de los sistemas de neurotransmisión del ácido $\gamma$-aminobutírico (GABA) y/o glicina o con menor frecuencia de la inhibición de los sistemas de neurotransmisión de aspartato y/o glutamato. Se presenta por exposición a benzodiacepinas, barbitúricos, anestésicos inhalatorios, hidrato de cloral, propofol, agentes antiepilépticos, etanol y otros alcoholes.

Al toxíndrome anticolinérgico también se le recuerda con la nemotecnia: caliente como liebre, rojo como remolacha, seco como hueso, ciego como murciélago y loco como sombrerero. Por su parte, el colinérgico cuenta con dos nemotecnias, una hace referencia a las manifestaciones muscarínicas y se denomina DUMBELS (D de diarrea y diaforesis, $U$ de incontinencia urinaria, $M$ de miosis, B de bradicardia, broncorrea y broncoespasmo, 
E de emesis, $L$ de lagrimeo y $S$ de sialorrea y crisis convulsivas); mientras que la otra hace referencia a las manifestaciones nicotínicas y consiste en los días de la semana en inglés ( $M$ de midriasis, $T$ de taquicardia e hipertensión, $\mathrm{W}$ de debilidad, $\mathrm{T}$ de temblor, $\mathrm{F}$ de fasciculaciones, $S$ de somnolencia y $S$ de crisis convulsivas). Finalmente, para el opioide suele considerarse la tríada: depresión del estado de alerta, depresión respiratoria y miosis $^{5-9,12,13}$.

\section{Otros datos clínicos que considerar}

Por otro lado, cabe aclarar que no es posible integrar un toxíndrome a partir de la mayoría de las sustancias que se conocen tóxicas debido a la amplia diversidad de mecanismos de acción que presentan. Por lo anterior, señalamos a continuación algunos aspectos clínicos a considerar en un paciente intoxicado.

- Estado neurológico. Con especial atención al estado de alerta, considerando que entre las causas toxicológicas del coma comúnmente encontramos involucradas sustancias de abuso (etanol, heroína, etc.), medicamentos (anestésicos, hipoglucemiantes orales, etc.), plantas (cannabis, peyote, etc.), con los cuales no se presentan datos de focalización por efecto directo y pueden diferenciarse de otras causas de deterioro del estado de alerta como enfermedad vascular cerebral y trauma craneoencefálico, pero sin dejar de lado los vasopresores, simpaticomiméticos o anticolinérgicos como posibles causas de un deterioro de la función neurológica focalizado $y$ asociado a isquemia o hemorragia. Las causas de agitación en el contexto toxicológico pueden estar dadas por simpaticomiméticos, anticolinérgicos 0 abstinencia a sustancias de abuso ${ }^{15}$. Las funciones mentales superiores (cálculo, juicio, memoria, etc.) pueden alterarse en el curso de una intoxicación por alucinógenos, pero también pueden estar alteradas por xenobióticos inductores de hipoxia (cianuro, monóxido de carbono, gases asfixiantes y metahemoglobinizantes, entre otros). Es el caso de los estados confusionales, que pueden estar dados por sobredosis de antidepresivos, antipsicóticos, ergotamínicos y no solo por abuso de sustancias. Entre los principales xenobióticos proconvulsivantes encontramos a los antidepresivos cíclicos, monóxido de carbono, hongos Gyromitra, isoniazida, anfetaminas y las abstinencias a sedantes-hipnóticos. Finalmente, la afección motora tiende a asociarse más comúnmente a antipsicóticos, litio, mercurio, colinérgicos e hidrocarburos, especialmente en su consumo crónico ${ }^{13,16,17}$.
- Pupilas. La presencia de miosis sugiere exposición a opioides, colinérgicos (efecto muscarínico) y clonidina, mientras que la midriasis sugiere exposición a anticolinérgicos (midriasis sin respuesta), simpaticomiméticos, abstinencia a opioides y colinérgicos (efecto nicotínico) $)^{5,9,13}$.

- Piel. En relación con el color podemos destacar la palidez por empleo de vasopresores (ergotamínicos, compuestos imidazólicos) o por hemólisis/depresión medular (plomo, platinoides, metotrexato); el color azul que se presenta en intoxicación por cianuro, metahemoglobinizantes, plata o amiodarona; el rojo en la intoxicación por monóxido de carbono, anticolinérgicos, vancomicina o disulfiram-etanol; el amarillo que puede asociarse a insuficiencia hepática (paracetamol, amanitas) o sin ella (betacarotenemia). En lo que respecta a la función glandular de la piel podemos encontrar diaforesis en la exposición a colinérgicos o asociada a hipoglucemia (hipoglucemiantes orales, insulina), y sequedad en el caso de los anticolinérgicos o asociada a pérdida hídrica (anfetaminas, diuréticos). Así mismo, deberá analizarse la piel en búsqueda de lesiones específicas (quemadura por cáusticos, dermatosis por níquel y huellas de mordedura de serpiente, entre otras) $)^{5,9,12,13}$.

- Aliento. En la intoxicación por etanol, el aliento presenta un olor sui generis, este olor se vuelve más dulce en la ingesta de otros alcoholes como isopropanol o etilenglicol; en el caso del cianuro se describe como olor a almendras amargas, el cual no es detectable por todas las personas; el olor a ajo se asocia a organofosforados, arsénico y fosfuros; la cicuta se presenta con olor a zanahoria; el hidrato de cloral a pera y los sulfuros con olor a huevo podrido $0^{5,9}$.

- Tórax. La dificultad respiratoria es común en intoxicación por organofosforados, carbamatos, mordedura por elápido, picadura de alacrán y especies marinas (pez piedra, pulpo de anillos azules, etc.). Cuando se acompaña de datos de neumonitis deben considerarse ingesta de hidrocarburos, aceite mineral y drogas de abuso inhalatorias (adulterantes); si se presenta con edema pulmonar o síntomas irritantes (dolor, tos, expectoración) deben considerarse: herbicidas, gases irritantes, vapor de metales (soldadores), cáusticos e inhalación de humo. Por otro lado, el ritmo cardiaco puede modificarse por la exposición a betabloqueadores, calcioantagonistas, lidocaína y otros antiarrítmicos, fenitoína, antidepresivos tricíclicos y anfetaminas, principalmente $\mathrm{e}^{5,9,12,13}$. 
Tabla 4. Xenobióticos potencialmente radiopacos*

\begin{tabular}{|l|l|l|l|}
\hline Ácido acetilsalicílico & Carbamazepina & Droga en paquetes & Nistatina \\
\hline Aluminio & Cesio & Espironolactona & Permanganato de potasio \\
\hline Amitriptilina & Clorpromazina & Haloperidol & Plomo \\
\hline Antimonio & Clorpropamida & Hidrato de cloral & Sales de potasio \\
\hline Arsénico & Cloruro de amonio & Hierro & Selenio \\
\hline Bario & Cloruro de metileno & Litio & Sucralfato \\
\hline Baterías & Cloruro de sodio & Medicamentos con capa entérica & Sulfato de zinc \\
\hline Bismuto & Cobalto & Mercurio & Talio \\
\hline Bromo & Cobre & Metotrexato & Tetracloroetileno \\
\hline Cadmio & Cromo & Neostigmina & Tetracloruro de carbono \\
\hline Calcio & Dietilestilbestrol & Níquel & Tricloroetileno \\
\hline
\end{tabular}

* La radioopacidad puede variar en función de la presentación, cantidad, concentración y el tiempo transcurrido desde la exposición. Adaptada de Savitt, et al., 198725; Florez, et al., 199826; Costello, et al., 200427.

- Abdomen. El hiperperistaltismo, ya sea que se presente o no con diarrea y cólicos puede observarse en intoxicación por organofosforados, carbamatos, detergentes, aceites esenciales, intoxicación alimentaria o catárticos (sulfato de magnesio, citrato de sodio, etc.). El íleo suele encontrarse en intoxicación por anticolinérgicos, opioides, mordedura por elápido y especies marinas, entre otros. Finalmente, a nivel abdominal debe descartarse la presencia de globo vesical, el cual está asociado con exposición a anticolinérgicos $5,9,12,13$.

\section{Complemento diagnóstico}

Las pruebas de laboratorio disponibles para el protocolo diagnóstico en el paciente intoxicado podemos clasificarlas en generales y específicas. Dentro de las pruebas generales encontramos aquellas habituales que nos orientan en relación con el estado general del paciente y que son susceptibles de modificarse ante la exposición a determinados agentes tóxicos. Estas pruebas incluyen electrolitos séricos, pruebas de funcionamiento hepático (aspartato aminotransferasa, alanina aminotransferasa, bilirrubinas, etc.), renal (creatinina, nitrógeno ureico en sangre, etc.), gasometría $(\mathrm{pH}$, bicarbonato, etc.) y citometría hemática (hemoglobina, plaquetas, etc.), entre las más importantes. En lo que respecta a las pruebas específicas podemos contar con aquellas cualitativas (perfil toxicológico en orina, prueba del cloruro férrico para salicilatos y prueba de ditionito para herbicidas bipiridilos), en ellas se determina una exposición únicamente como «positiva» o «negativa» sin establecer valores de referencia. Finalmente, otro tipo de pruebas específicas son las cuantitativas (concentración sérica de paracetamol, carbamazepina, ácido valproico, etanol y porcentaje de carboxihemoglobina, entre otras), las cuales a partir de una escala $(\mathrm{mg} / \mathrm{dl}$, $\mathrm{mmol} / \mathrm{l}$, etc.) nos permiten estadificar las exposiciones a diversos xenobióticos. En este sentido, conviene familiarizarse con las pruebas que se encuentran disponibles en la unidad médica de que se trate ${ }^{18-22}$.

Como parte del complemento diagnóstico los estudios de imagen representan una herramienta útil en el abordaje del paciente intoxicado. Entre ellos encontramos las técnicas de radioimagen y en menor medida los estudios de radioisótopos (biomarcadores), que de manera directa 0 indirecta contribuyen al diagnóstico de las intoxicaciones ${ }^{23}$. La identificación directa se lleva a cabo cuando el xenobiótico (por sí mismo o por su envase) es observable mediante la técnica utilizada, tal como sucede con algunos xenobióticos como los metales, cuerpos extraños, paquetes de drogas, iones, fármacos de liberación prolongada o bien aquellos que forman bezoares que pueden observarse en las radiografías simples ${ }^{24-26}$ (Tabla 4); mientras que la identificación indirecta hace referencia a la conjunción de los hallazgos obtenidos en determinado estudio, como en el caso de los datos de perforación esofágica en la ingestión de cáusticos (aire libre subdiafragmático, enfisema subcutáneo o neumopericardio, además de otros hallazgos en la telerradiografía de tórax) o la lesión de 
ganglios basales en la intoxicación por monóxido de carbono (hipodensidad a nivel de globo pálido en una tomografía de cráneo en fase simple) ${ }^{27}$. No obstante, son limitados los casos de exposición a xenobióticos que requieren un estudio de imagen como parte del complemento diagnóstico, por lo que su solicitud deberá estar precedida de un adecuado interrogatorio y exploración física que lo justifiquen.

\section{Agradecimientos}

Agradecemos a la Dra. Janett Santos Sánchez por haber sentado las bases para la formación física y académica de este centro toxicológico para quienes hoy laboramos en él.

\section{Financiamiento}

El presente artículo no ha recibido ninguna beca especifica de agencias de los sectores públicos, comercial o sin ánimos de lucro.

\section{Conflicto de intereses}

Los autores declaran no tener conflicto de intereses.

\section{Responsabilidades éticas}

Protección de personas y animales. Los autores declaran que para esta investigación no se han realizado experimentos en seres humanos ni en animales.

Confidencialidad de los datos. Los autores declaran que en este artículo no aparecen datos de pacientes.

Derecho a la privacidad y consentimiento informado. Los autores declaran que en este artículo no aparecen datos de pacientes.

\section{Bibliografía}

1. Thomas SHL, Lewis S, Bevan L, Bhattacharyya MG, Bramble K, Chew J, et al. Factors affecting hospital admission and length of stay of poisoned patients in the North East of England. Hum Exp Toxicol. 1996;15(11):915-9.

2. Strotmeyer SJ, Weiss HB. Injuries in Pennsylvania: hospital discharges, 1999. Pittsburgh: Center for injury research and control, Department of Neurologic Surgery, University of Pittsburgh; 2000.
3. Khare MM, Bhide MM, Ranade AA, Jaykar AA, Paniker LL, Patnekar PN. Poisoning in children-analysis of 250 cases. J Posgrad Med. 1990;36(4):203-6.

4. Donovan JW, Burkhart K, Brent J. The critically poisoned patient. En: Brent J. Critical care toxicology. Diagnosis and management of the critically poisoned patient. $2^{\text {nd }}$ ed. EE.UU.: Springer; 2017. pp. 3-27.

5. Erickson TB, Thompson TM, Lu JJ. The approach to the patient with an unknown overdose. Emerg Med Clin N Am. 2007;25(2):249-81.

6. Levine M, Brooks DE, Truitt CA, Wolk BJ, Boyer EW, Ruha AM. Toxicology in the ICU. Part 1: General overview and approach to treatment. Chest. 2011;140(3):795-806.

7. Lam SW, Engebretsen KM, Bauer SR. Toxicology today: what you need to know now. J Pharm Pract. 2011;24(2):174-88.

8. Mokhlesi B, Leiken JB, Murray P, Corbridge TC. Adult toxicology in critical care: part 1: general approach to the intoxicated patient. Chest. 2003;123(2):577-92.

9. Nelson LS, Howland MA, Lewin NA, Smith SW, Goldfrank LR, Hoffman RS. Initial evaluation of the patient: Vital signs and toxic syndromes. En: Nelson LS, Howland RS, Howland MA, Lewin NA, Goldfrank LR, Smith SW, editores. Goldfrank's toxicologic emergencies. 11 $1^{\text {th }}$ ed. EE.UU.: Mc Graw-Hill; 2019. pp. 28-31.

10. Merchant RM, Topjian AA, Panchal AR, Cheng A, Aziz K, Berg KM, et al. 2020 American Heart Association guidelines for cardiopulmonary resuscitation and emergency cardiovascular care. Circulation 2010; 142:S337-S357.

11. Disque K. Pediatric Advanced Life Support (PALS). Satori Continuum Publishing; 2016.

12. Erickson TB, Aks S, Gussow L, Williams RH. Toxicology update: a rational approach to managing the poisoned patient. Emerg Med Pract. 2001;3(8):1-28.

13. Pizon AF, Yanta JH, Swartzentruber GS. The diagnostic process in medical toxicology. En: Brent J, editor. Critical care toxicology. Diagnosis and management of the critically poisoned patient. $2^{\text {nd }}$ ed. EE.UU.: Springer; 2017. pp. 29-41.

14. Mofenson HC, Greensher J. The unknown poison. Pediatrics. 1974;54(3):336-42.

15. Young GP. The agitated patient in the emergency department. Emerg Med Clin North Am. 1987:5(4):765-81.

16. Delaney KA, Kolecki P. Central nervous system depression. En: Ford MD, Delaney KA, Ling LJ, Erickson TB. Clinical toxicology. $1^{\text {st }}$ ed. EE.UU.: W.B. Saunders Company; 2001. pp. 137-145.

17. Merigian KS, Hedges JR, Roberts JR, Childress RA, Niehaus MA, Franklin N. Use of abbreviated mental status examination in the initial assessment of overdose patients. Arch Emerg Med. 1988;5(3):139-45.

18. Grunbaum AM, Rainey PM. Laboratory principles. En: Nelson LS Howland RS, Howland MA, Lewin NA, Goldfrank LR, Smith SW, editores. Goldfrank's toxicologic emergencies. 11 $1^{\text {th }}$ ed. EE.UU.: Mc Graw-Hill; 2019. pp. 101-113.

19. Taylor RL, Cohan SL, White JD. Comprehensive toxicology screening in the emergency department: an aid to clinical diagnosis. Am J Emerg Med. 1985;3(6):507-11.

20. Brett AS. Implications of discordance between clinical impression and toxicology analysis in drug overdose. Arch Intern Med. 1988;148(2):437-41.

21. Belson MG, Simon HK. Utility of comprehensive toxicologic screens in children. Am J Emerg Med. 1999;17(3):221-4.

22. Kellermann AL, Finn SD, LoGerfo JP, Copass MK. Utilization and yield of drug screening in the emergency department. Am J Emerg Med. 1988:6(1):14-20.

23. Schwartz DT. Principles of diagnostic imaging. En: Nelson LS, Howland RS, Howland MA, Lewin NA, Goldfrank LR, Smith SW, editores. Goldfrank's toxicologic emergencies. $11^{\text {th }}$ ed. EE.UU.: Mc Graw-Hill; 2019. pp. 114-139.

24. Jones JS, Lagasse J, Zimmerman G. Computed tomographic findings after acute carbon monoxide poisoning. Am J Emerg Med. 1994;12(4):448-51.

25. Savitt DL, Hawkins HH, Roberts JR. The radiopacity of ingested medications. Ann Emerg Med. 1987;16(3):331-39.

26. Florez MV, Evans JM, Daly TR. The radiodensity of medications seen on x-ray films. Mayo Clin Proc. 1998;73(6):516-9.

27. Costello J, Townend W. Best evidence topic report. Abdominal radiography in "body packers". Emerg Med J. 2004;21(4):498. 